predictive biomarkers of response to anti-epidermal growth factor receptor (EGFR) inhibition. KRAS protein activity requires specific binding to the lipid plasma membrane; the association between serum lipid profile and KRAS status has not so far been evaluated.

Methods A total of 201 patients with colorectal cancer stage IV, diagnosed between January 2012 and July 2017, were retrospectively analysed. Data including KRAS status, cancer location, total serum cholesterol, cholesterol:HDL ratio, LDLcholesterol, HDL-cholesterol and tryglicerides were collected from clinical records. We used chi-square test to evaluate associations between serum lipid profile and KRAS status in stage IV colorectal cancer patients.

Results Out of 201 metastatic colorectal cancer patients 122 $(60.69 \%)$ were males and $79(39.30 \%)$ were females (mean age 65.38 , standard deviation 11.39). Eighty-two (40.79\%) patients exhibited KRAS mutation and 119 (59.20\%) KRAS wild type. Ninety-five patients $(47.26 \%)$ had cancer located at ascending colon, transverse colon and splenic flexure and 106 $(52.73 \%)$ patients had cancer located at sigmoid colon and rectum. There was a significant association between KRAS mutation and high cholesterol:HDL ratio in colon cancer located at sigmoid colon and rectum [odds ratio $(\mathrm{OR})=3.09$, $95 \%$ confidence interval (CI) $1.3-7.0, \mathrm{p}=0006]$, but not in colon cancer located at ascending colon, transverse colon and splenic flexure $(\mathrm{OR}=0.76,95 \%$ CI $0.27-1.39, \mathrm{p}=0.24)$.

Conclusion Our results reveal an association between high cholesterol:HDL ratio and KRAS mutation in sigmoid and rectal cancer. Specific tumour location and lipid profile may be useful in prognostic and predictive stratification of clinical outcomes.

\section{PTU-066 THE GUT MICROBIOTA INFLUENCES INTESTINAL EPITHELIAL PROLIFERATIVE POTENTIAL}

${ }^{1}$ John P Thomas*, ${ }^{2}$ Aimee Parker, ${ }^{2}$ Devina Divekar, ${ }^{2}$ Carmen Pin, ${ }^{3}$ Alastair Watson. ${ }^{1}$ Department of Gastroenterology, Norfolk and Norwich University Hospital, Norwich, UK; ${ }^{2}$ Gut Health and Food Safety Research Programme, Institute of Food Research, Norwich, UK; ${ }^{3}$ Norwich Medical School, University of East Anglia, Norwich, UK

\subsection{6/gutjnl-2018-BSGAbstracts.407}

Introduction The intestinal epithelium is comprised of a single layer of cells which serves a number of critical functions including the formation of a physical barrier to environmental pathogens and chemical substances, and the absorption of essential nutrients, electrolytes and water. It is also the site of the gut microbiota, a complex and diverse community of bacteria, viruses and fungi, which exists in a mutually beneficial relationship with the human host. The epithelial barrier is maintained through tightly regulated processes of stem cell renewal, epithelial maturation, cell migration and cell death. Failure to finely coordinate these processes can lead to disease states such as cancer. In this study, we aimed to investigate and characterise the role of the intestinal microbiota on epithelial cell proliferation.

Methods We determined the rates of epithelial proliferation in the intestines of Specific-Pathogen-Free (SPF) mice and GermFree (GF) mice. We utilised a previously described method which integrates cell tracking using the thymidine analogue Bromodeoxyuridine (BrdU) in crypt-villus units, with a tailored mathematical model, to assess the spatiotemporal dynamics of epithelial cell behaviour in SPF and GF conditions.
Results The rate of epithelial cell production in GF conditions was significantly slower in the colon, ileum and jejunum in comparison to SPF conditions. In the duodenum, there were no significant differences in proliferation rates in GF and SPF conditions. Cell production rates progressively decreased towards the distal part of the intestine, which inversely correlate with the concentration of organisms constituting the intestinal microbiota.

Conclusions These findings indicate that the gut microbiota plays an important role in determining intestinal epithelial cell proliferation rates. This relationship may have important implications in conditions such as colorectal cancer and inflammatory bowel disease, where differences in microbial signatures are known to exist. In turn, it may be possible to harness this knowledge to alter disease progression by modifying the host microbiota.

\section{PTU-067 THE POSITIVE IMPACT OF THE BOWEL CANCER SCREENING PROGRAMME ON COLORECTAL CANCER DIAGNOSES AND OUTCOMES}

Ajay Verma*, Andrew Chilton. Department of Gastroenterology, Kettering General Hospital NHS foundation trust, Kettering, UK

10.1136/gutjnl-2018-BSGAbstracts.408

Introduction The impact of bowel cancer screening programmes (BCSP) on down-staging colorectal cancer (CRC) at presentation is well established and national screening is largely thought to be a success. Uptake of screening remains less than $60 \%$, screening age has been expanded to ages 60 75 and Bowel Scope screening is being rolled out.

Despite BCSP, the number of UK CRC cases has increased by approximately $5 \%$ in the last decade. We aim to evaluate the impact of BCSP on the stage of colorectal cancer cases at presentation.

Methods Between 2013 \& 2016, approximately 700 new cases of CRC were discussed at the colorectal cancer multi-disciplinary team (MDT) meeting at Kettering General Hospital. The BCSP screening practitioners have collated demographic data, CRC stage, engagement with BCSP (at any time including prior FOBt negative return), emergency presentation, and whether surgery was performed open or laparoscopically.

681 cases have had a full dataset collated and analysed, we report on this data. It is noted that elderly patients over 70 may not have received BCSP invite (though are able to opt in), patients under 55 are not eligible for BCSP (a small number of cases).

Results In 681 analysed cases, 306 patients (44.9\%) had engaged with a BCSP invite (previously and/or at time of diagnosis), 375 had not (55.1\%), p $\leq \mathbf{0 . 0 1}$.

\begin{tabular}{llll}
\multicolumn{2}{l}{ Abstract PTU-067 Table 1 } & & \\
\hline $\begin{array}{l}\text { Dukes' } \\
\text { stage }\end{array}$ & BCSP & non-BCSP & Total \\
\hline A & $86(28.1 \%)$ & $47(12.5 \%)$ & 133 \\
B & $91(29.7 \%)$ & $120(32.0 \%)$ & 211 \\
C & $116(37.9 \%)$ & $173(46.1 \%)$ & 289 \\
D & $13(4.3 \%)$ & $35(9.3 \%)$ & 48 \\
All cases & 306 & 375 & 681 \\
& $105 \% 201$ & $170 \% 205$ & \\
& $\delta$ & $\delta$ & \\
\hline
\end{tabular}

